\title{
Application de l'intensimétrie acoustique à l'identification des sources de pulsation de pression dans des circuits
}

\author{
Use of acoustic intensimetry for identifying \\ pressure pulse sources in piping systems
}

\author{
A. Badie-Cassagnet, M. Bockhoff, J.M. Lambert * \\ Centre technique des industries mécaniques (CETIM)
}

\section{Introduction}

En acoustique industrielle on est fréquemment amené à étudier le problème de la propagation d'ondes acoustiques dans un conduit connecté à une (ou même plusieurs) source(s) de pulsation de pression: pompe, débitant dans un circuit de liquide (eau, huile,...), compresseur ou ventilateur, alimentant un conduit de gaz, etc... La vitesse d'écoulement y est généralement suffisamment faible pour être négligeable devant la célérité du son c. Par ailleurs, les fréquences d'excitation rencontrées dans la plupart des problèmes industriels sont très inférieures aux fréquences de coupure des conduits (notamment lorsqu'il s'agit de liquides) c'està-dire l'essentiel de l'énergie acoustique est transporté sous forme d'ondes planes.

Dans ces conduits on observe souvent des phénomènes d'ondes quasi-stationnaires, dûs aux réflexions des ondes acoustiques sur des obstacles tel que dia. phragmes, vannes ou coudes de faible rayon de courbure. L'amplitude $p(x, t)$ des fluctuations de pression, pour une fréquence $f$ donnée, varie alors beaucoup avec la position dans le conduit. Lorsque le bruit émis par la source contient des sons purs, l'estimation du niveau moyen des pulsations de pression nécessiterait en théorie l'implantation d'un grand nombre de capteurs disposés tout le long du conduit, ou encore l'utilisation d'une sonde mobile. Ces deux techniques ne sont pas réalistes dans les installations industrielles.

Nous allons montrer que l'information recueillie par deux capteurs seulement est souvent suffisante pour caractériser complétement une onde quasi-stationnaire, à condition de mesurer, en plus des niveaux de pression, le déphasage entre les signaux.

* actuellement : Laboratoire National d'Essais, 1, rue G. Boissier, 75015 Paris.

\section{L'onde quasi-stationnaire}

Considérons une onde plane d'amplitude $p_{0}$, de fréquence $f=\omega / 2 \pi$ et de nombre d'onde $k=\omega / c$ se propageant dans la direction $-x$ et frappant à $x=0$ une paroi caractérisée par un coefficient de réflexion $r=r_{0} e^{i \gamma}$. La pression acoustique dans l'onde quasi-stationnaire qui résulte de la superposition des ondes incidentes et réfléchies est donnée par:

$$
p(x, t)=p_{0} e^{i \omega t}\left(e^{-i k x}+r_{0} e^{i(k x+\gamma)}\right)
$$

et l'équation d'Euler permet d'en déduire la vitesse acoustique :

$u_{x}(x, t)=-\frac{1}{\rho} \int \frac{\partial p}{\partial x} d t=\frac{p_{0}}{\rho c} e^{i \omega t}\left(e^{-i k x}-r_{0} e^{i(k x+\gamma)}\right)$

( $\rho:$ masse volumique du fluide)

L'intensité apparente qu'on mesure habituellement est donnée par la valeur quadratique moyenne de la pression :

$I_{0}(x)=\frac{\overline{p^{2}}}{\rho c}=\frac{p_{0}^{2}}{2 \rho c}\left(1+r_{0}^{2}+2 r_{0} \cos (2 k x+\gamma)\right)$

Cette quantité présente une succession de maxima (ventre de $p$ ) et de minima (nœuds de $p$ ) séparés par la distance $\lambda / 4(\lambda$ : longueur d'onde $)$ :

$I_{0 \max }=\frac{p_{0}^{2}}{2 \rho c}\left(1+r_{0}\right)^{2}$ et $I_{0 \min }=\frac{p_{0}^{2}}{2 \rho c}\left(1-r_{0}\right)^{2}(4)$

On appelle taux d'onde progressive $\beta$, le rapport:

$$
\beta=\left(\frac{I_{0 \min }}{I_{0 \max }}\right)^{\frac{1}{2}}=\frac{1-r_{0}}{1+r_{0}}
$$

$\beta=1$ correspond à une onde progressive, $\beta=0$ à une onde purement stationnaire. 
Le vecteur intensité acoustique, défini par $\vec{I}=\frac{1}{2} \operatorname{Repu} *$

(* désigne le complexe conjugué) se réduit dans le cas présent à la seule composante

$$
I_{x}=\frac{1}{2} \operatorname{Repu}_{x}^{*}=\frac{p_{0}^{2}}{2 \rho c}\left(1-r_{0}^{2}\right)
$$

Contrairement à l'amplitude des fluctuations de pression, le vecteur intensité est constant tout le long de l'onde quasi-stationnaire, c'est-à-dire qu'il n'y a ni absorption, ni création d'énergie dans le fluide. Notons encore deux relations entre l'intensité $I_{x}$ et intensités apparentes :

$$
I_{0 \max }=\frac{1}{\beta} I_{x} \quad \text { et } \quad I_{0 \min }=\beta I_{x}
$$

Les grandeurs $I_{x}$ et $\beta$ définissent alors complétement l'onde quasi-stationnaire, $I_{x}$ fixant le niveau et $\beta$ caractérisant l'écart entre les maxima et les minima de la pression acoustique.

\section{Caractérisation d'une onde quasi-stationnaire par intensimètre}

\subsection{Détermination de l'intensité par la méthode des différences finies}

Nous nous limitons ici à quelques notions élémentaires, des études plus détaillées ayant déjà été publiées, sur la méthode elle-même, [1] à [4], et sur certains aspects de son utilisation pour des mesures en conduit, $[5],[6]$.

Le principe de cette méthode consiste à considérer les pressions acoustiques $p_{1}\left(x_{1}, t\right)$ et $p_{2}\left(x_{2}, t\right)$ en deux points d'une onde quasi-stationnaire et d'approcher dans relation (2) l'expression $\frac{\partial p}{\partial x} \operatorname{par} \frac{\Delta p}{\Delta x}=\frac{p_{1}-p_{2}}{x_{1}-x_{2}}$.

On montre alors qu'une approximation $\widetilde{I}_{x_{0}}$ de la valeur exacte $I_{x_{0}}$ est obtenue par la relation:

$$
\tilde{I}_{x_{0}}=\frac{1}{2 \rho c} \frac{\operatorname{Im} p_{1} p_{2}^{*}}{k \Delta x} ; x_{0}=\frac{x_{1}+x_{2}}{2}
$$

Le rapport entre $\widetilde{I}_{x_{0}}$ et $I_{x_{0}}$ étant donné par $\widetilde{I}_{x_{0}}=$ $I_{x_{0}} \frac{\sin k \Delta x}{k \Delta x}$, on peut aussi écrire :

$$
I_{x_{0}}=\frac{1}{2 \rho c} \frac{\operatorname{Im} p_{1} p_{2}^{*}}{\sin k \Delta x}=\frac{p_{0_{1}} p_{0_{2}}}{2 \rho c} \frac{\sin \Delta \varphi}{\sin k \Delta x}
$$

$\Delta \varphi$ : déphasage entre les pressions $p_{1}$ et $p_{2}$.

Cette relation montre que pour un son pur l'intensité acoustique peut être déterminée en mesurant les amplitudes $p_{0_{1}}$ et $p_{0_{2}}$ (à l'aide de capteurs de pression) et le déphasage $\Delta \varphi$ entre les deux signaux (à l'aide d'un phasemètre), les paramètres $\Delta x, f, c$ étant supposé connus.

Lorsque le bruit couvre une large bande de fréquences, il est nécessaire d'effectuer une analyse de Fourier simultanée des signaux issus des deux capteurs et de calculer le spectre croisé $S_{21}(f)=X_{1}(f) X_{2}^{*}(f)$ qui est le produit de la transformée de Fourier du signal 1 par celle du signal 2 conjuguée.

Il en résulte une expression pour l'intensité analogue à l'équation (8):

$$
I_{x_{0}}(f)=\frac{1}{2 \rho c} \frac{\operatorname{Im} S_{21}(f)}{\sin k \Delta x}
$$

La mesure d'intensité acoustique par cette technique présente l'avantage d'être indépendante de la position des capteurs le long du conduit. Le résultat d'une telle mesure peut être soit positif, soit négatif (suivant le signe de $\Delta \varphi$ ) et indique le sens de propagation de l'énergie acoustique.

Dans le cas où plusieurs sources sont connectées au conduit, la mesure d'intensité acoustique donne la somme algébrique des intensités créées par chaque source et il peut y avoir compensation conduisant à une intensité très faible, alors que les niveaux de pression sont élevés.

Toutefois, il est assez rare que deux sources situées en amont et aval du point d'observation produisent des intensités égales. En général l'une l'emporte nettement sur l'autre à certaines fréquences, l'inverse se produisant à d'autres fréquences. Le signe de la fonction $I_{x}(f)$ permet alors de séparer les deux sources et de déterminer les fréquences pour lesquelles chacune est prépondérante. Les fréquences pour lesquelles la compensation s'effectue peuvent être repérées par une chute de la fonction de cohérence entre les signaux due au fait que le déphasage $\Delta \varphi$ prend des valeurs aléatoires.

\subsection{Etude du déphasage entre les pressions en deux points}

La mesure d'intensité acoustique ne permet pas de caractériser complètement une onde stationnaire. Elle ne donne d'information ni sur la position des ventres et des nouds le long du conduit, ni sur la valeur du taux d'onde progressive, mais ces renseignements peuvent être obtenus par l'étude du déphasage $\Delta \varphi(f)$.

Le déphasage entre $p_{1}$ et $p_{2}$ est donné par:

$$
\operatorname{tg} \Delta \varphi=\operatorname{Arg} p_{1} p_{2}^{*}
$$

En utilisant pour $p_{1}$ et $p_{2}$ des représentations de la forme d'équation (1) et en tenant compte de la définition du taux d'onde progressive (Equ. (5)), on obtient:

$$
\operatorname{tg} \Delta \varphi=\frac{2 \beta \sin k \Delta x}{\left(1+\beta^{2}\right) \cos k \Delta x+\left(1-\beta^{2}\right) \cos \left(2 k x_{0}+\gamma\right)}
$$

Le déphasage dépend donc de $\beta, \Delta x, x_{0}$ et $f$. Deux cas particuliers peuvent se présenter:

$$
f=\text { constant : }
$$

Pour une fréquence fixée et un écartement de capteurs fixé, le déphasage varie en fonction de la position médiane $x_{0}$ des capteurs. Il est minimum aux ventres de pression et maximum aux nœuds:

$$
\operatorname{tg} \frac{\Delta \varphi_{\min }}{2}=\beta \operatorname{tg} \frac{k \Delta x}{2} \text { et } \operatorname{tg} \frac{\Delta \varphi_{\max }}{2}=\frac{1}{\beta} \operatorname{tg} \frac{k \Delta x}{2}
$$


L'amplitude des variations du déphasage est essentiellement liée aux taux $\beta$, elle est nulle lorsque l'onde est progressive $(\beta=1)$ et augmente si l'onde devient plus stationnaire.

L'obtention expérimentale de $\Delta \varphi$ en fonction de $x_{0}$ suppose que l'on réalise des mesures en déplaçant l'ensemble des deux capteurs dans l'onde. Ceci n'est en général pas pratique et rend cette approche d'un intérêt très limité.

\section{$x_{0}=$ constant $:$}

Par contre dans le cas plus réaliste d'une seule position de mesure $x_{0}$ (souvent l'insertion des capteurs n'est possible qu'à un endroit) et lorsque le bruit est à large bande il est plus facile d'étudier le déphasage en fonction de la fréquence qui est alors :

$$
\Delta \varphi(f)=\operatorname{Arg} S_{21}(f)
$$

Cette courbe dépend $\cdot$ du taux d'onde progressive $\beta$ - de la distance $x_{0}$ entre le centre des capteurs et le réflecteur

- de la célérité du son $c$.

Son étude permet de déterminer ces trois quantités. Nous nous limitons ici aux deux premières:

\subsubsection{Détermination du taux d'onde progressive $\beta$}

La courbe $\Delta \varphi(f)$ présente une succession de maxima et de minima correspondant au fait que le centre des capteurs est un nœud ou un ventre de pression. En utilisant les relations (11) $\beta$ peut être calculé à partir des valeurs maximales et minimales de $\Delta \varphi$ :

$$
\beta=\frac{\operatorname{tg} \frac{\Delta \varphi_{\text {min }}}{2}}{\operatorname{tg} \frac{k \Delta x}{2}} \text { et } \frac{1}{\beta}=\frac{\operatorname{tg} \frac{\Delta \varphi_{\text {max }}}{2}}{\operatorname{tg} \frac{k \Delta x}{2}}
$$

\subsubsection{Détermination de la distance $x_{0}$ à la paroi réflé- chissante}

Connaissant la célérité du son $c, x_{0}$ peut être calculé à partir de l'écart entre deux maxima ou deux minima de $\Delta \varphi(f)$ qui est de:

$$
\Delta f=\frac{c}{2 x_{0}}
$$

Ceci suppose que la paroi réfléchissante est unique quelle que soit la fréquence. On observe souvent des phénomènes plus complexes (réflexions sur plusieurs obstacles successifs par exemple); dans ce cas la succession des maxima et minima de $\Delta \varphi(f)$ n'est pas toujours régulière et l'interprétation devient plus difficile.

\section{Expériences}

\subsection{Mesures dans un tube de Kundt}

Le tube utilisé a un diamètre intérieur de $10 \mathrm{~cm}$ et une longueur de $1 \mathrm{~m}$. Il permet l'étude des ondes quasi- stationnaires jusqu'à $2 \mathrm{kHz}$ environ. Au fond du tube, contre la paroi réfléchissante a été placé un matériau absorbant, ce qui augmente le taux d'onde progressive $\beta$ surtout en hautes fréquences. Les capteurs utilisés sont des microphones miniatures mesurant la pression acoustique à une distance $\Delta x=12 \mathrm{~mm}$.

\section{Sons purs :}

Le but de l'expérience est de montrer que l'intensité mesurée est constante lorsqu'on déplace les capteurs le long de l'onde.

Pour cela on a mesuré les niveaux apparents $I_{0_{1}}$ et $I_{0_{2}}$ ainsi que le déphasage $\Delta \varphi$ en fonction de la distance $x_{0}$ entre capteurs et paroi réfléchissante.

La figure 1 montre pour deux fréquences les résultats des mesures ainsi que les valeurs calculées de l'intensité $I_{x}$. On constate une petite différence dans la mesure des niveaux pour chaque capteur qui peut être attribuée au phénomène de diffraction par les microphones. On a vérifié que cette dissymétrie ne provenait pas d'une différence entre les réponses des microphones en les échangeant. La conséquence est que l'intensité calculée n'est pas rigoureusement constante le long de l'onde. Sa variation atteint $2 \mathrm{~dB}$ pour $500 \mathrm{~Hz}$ et $0.5 \mathrm{~dB}$ pour $1000 \mathrm{~Hz}$

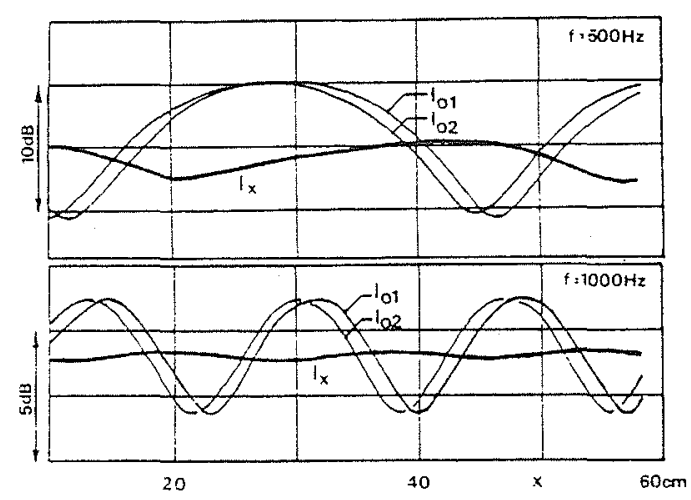

Figure 1. $-I_{0_{1}}, I_{0_{2}}$ et $I_{x}$ en fonction de $x_{0}$ pour les fréquences 500 et $1000 \mathrm{~Hz}$ (Tube de Kundt, sons purs)

\section{Bruit large bande :}

Le haut-parleur placé à l'extrémité du tube a été excité avec un bruit blanc couvrant la gamme $0-$ $2500 \mathrm{~Hz}$. Les microphones disposés comme dans l'expérience précédente sont placés à la distance $x_{0}=0.6 \mathrm{~m}$ de la paroi réfléchissance. On a enregistré les signaux délivrés par les deux microphones, le dépouillement effectué sur calculateur a permis d'obtenir le déphasage $\Delta \varphi$ en fonction de la fréquence: figure 2 (en haut).

A partir des maxima et minima de cette courbe, on a calculé le taux d'onde progressive $\beta$ (Eq. $\left(11^{\prime}\right)$ ) et comparé les résultats avec ceux obtenus de façon classique, c'est-à-dire fréquence par fréquence, à partir des intensités apparentes aux ventres et aux nœuds (Eq. $(5)$ ) : figure 2 (en bas).

Lorsque $\beta$ varie peu avec la fréquence, une assez bonne approximation peut être obtenue en faisant la moyenne des valeurs calculées à partir des équations $\left(11^{\prime}\right)$. 

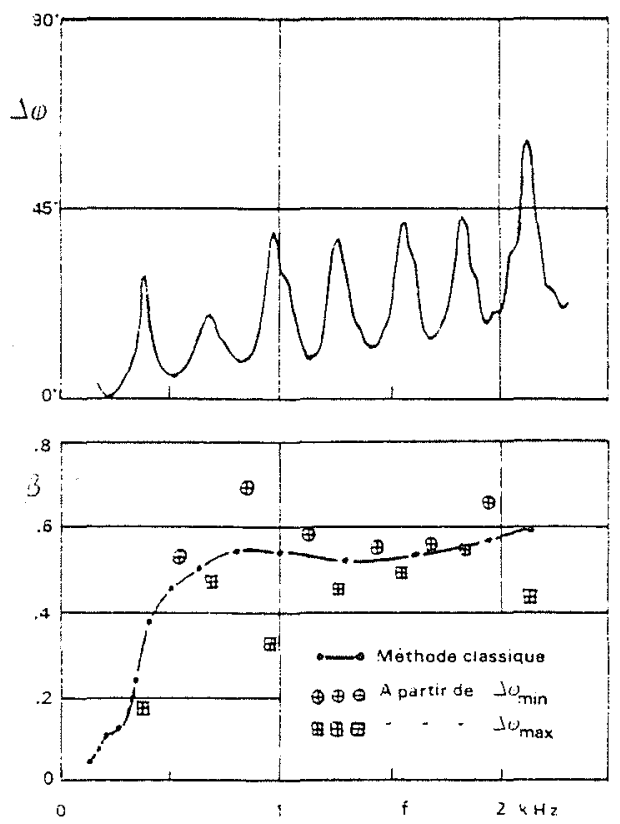

Figure 2. - Déphasage $\Delta \varphi$ et taux d'onde progressive $\beta$ en fonction de la fréquence $\cdot x_{0}=0.6 \mathrm{~m}$. (Tube de Kundt, bruit large bande)

\subsection{Mesures dans un circuit de transmission hydrau- lique (huile)}

On a réalisé des mesures à l'aide de deux capteurs de pression dans une canalisation contenant de l'huile sous pression statique de 25 bars. Les capteurs sont distants de $\Delta x=295 \mathrm{~mm}$ et disposés de façon à ce que leur membrane affleure la paroi intérieure de la canalisation. Celle-ci a un diamètre intérieur de $10 \mathrm{~mm}$ et comporte deux parties rectilignes d'une longueur de $6 \mathrm{~m}$ environ reliées par un coude: figure 3 . Une des extrémités est fermée presque totalement par une vanne et réfléchit les ondes générées par une servo-valve placée à l'autre extrémité. La servo-valve est excitée en son pur, un balayage manuel étant réalisé pour des fréquences comprises entre 100 et $600 \mathrm{~Hz}$. Le niveau d'excitation est ajusté pour que le niveau d'intensité reste constant quelque soit la fréquence.

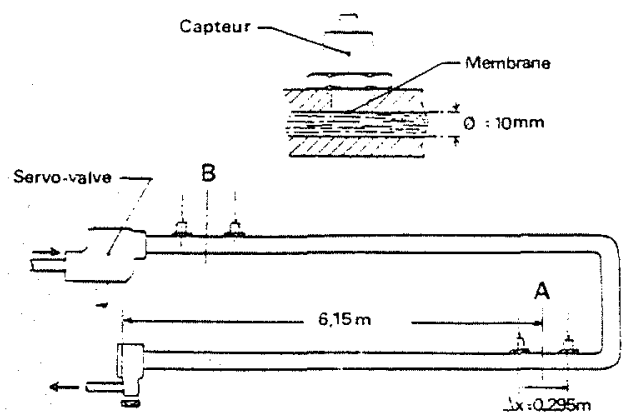

Figure 3. - Schéma de la canalisation d'huile

On a porté, figures 4 et 5 , les intensités apparentes $I_{0_{1}}(f)$ et $I_{0_{2}}(f)$, le déphasage $\Delta \varphi(f)$ ainsi que l'intensité calculée $I_{x}(f)$.
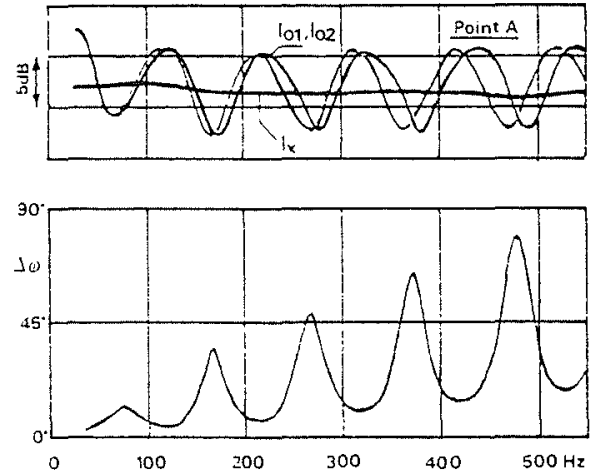

Figure 4. $-I_{0_{1}}, I_{0_{2}}, I_{x}$ et $\Delta \varphi$ en fonction de la fréquence au point A. (Canalisation d'huile)
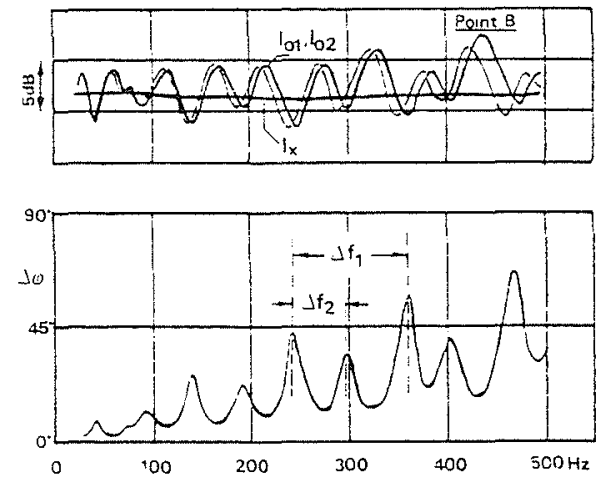

Figure 5. $-I_{0_{1}}, I_{0_{2}}, I_{x}$ et $\Delta \varphi$ en fonction de la fréquence au point $B$.

\section{Mesures au point $\mathbf{A}$ :}

L'évolution du déphasage $\Delta \varphi(f)$ avec la fréquence présente des maxima et minima séparés de $\Delta f=106 \mathrm{~Hz}$. La vitesse du son dans l'huile à la pression de 25 bar étant de $c=1300 \mathrm{~m} / \mathrm{s}$ environ, on déduit de l'équation (12) une distance théorique au réflecteur de $x_{0}=6.3 \mathrm{~m}$ qui en effet correspond à peu près à la distance réelle capteurs-extrémité.

Le calcul du taux d'onde progressive à partir des intensités apparentes (Equ. (5)) donne une valeur sensiblement indépendante de la fréquence égale à 0.38 . Les valeurs obtenues à partir de $\Delta \varphi_{\max }$ et $\Delta \varphi_{\min }$ sont de 0.41 et 0.36 respectivement, et leur moyenne est en bon accord avec la première. Cette valeur élevée est la conséquence d'une fluctuation peu importante des intensités apparentes $( \pm 5 \mathrm{~dB})$. Elle signifie que la vanne a un coefficient de réflexion assez faible, transmettant ainsi une grande partie de l'énergie incidente dans la canalisation située en aval.

\section{Mesures au point B :}

Ici on distingue deux écarts différents entre maxima de $\Delta \varphi: \Delta f_{1}=105 \mathrm{~Hz}$ et $\Delta f_{2}=53 \mathrm{~Hz}$ correspondant à des réflecteurs situés à $x_{0_{1}}=6,2 \mathrm{~m}$ (coude) et $x_{0_{2}}=$ $12,4 \mathrm{~m}$ (extrémité de la canalisation) respectivement.

Dans cette branche de la canalisation, on observe alors la superposition de deux ondes quasi-stationnaires et la succession de maxima et de minima de $\Delta(f)$ ne correspond plus à une valeur unique de $\beta$. En effet, une fois sur deux le rapport entre maxima et minima se réduit, traduisant un taux d'onde progressive plus grand dû à la réflexion plus faible sur le coude. 


\subsection{Mesures dans un circuit hydraulique industriel (eau)}

Les mesures ont été effectuées sur un circuit hydraulique reliant une pompe centrifuge à une vanne de régulation (servant de charge) par une tuyauterie de diamètre $150 \mathrm{~mm}$, voir figure 6 .

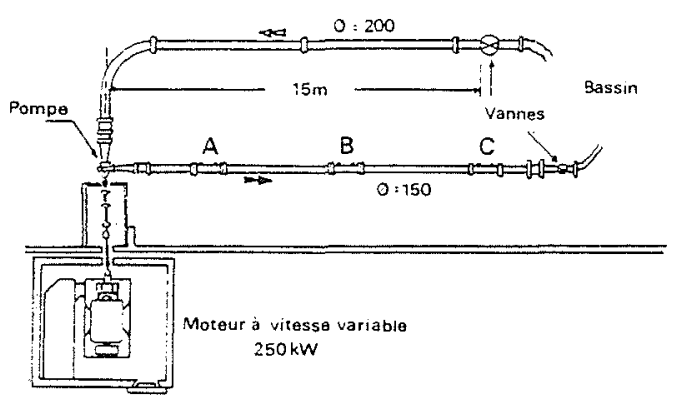

Figure 6. - Schéma du circuit hydraulique.

Trois couples de capteurs de pression à membranes affleurantes $(\Delta x=650 \mathrm{~mm})$ sont disposées sur des manchettes de tuyauterie pour observer le long du circuit l'évolution des pulsations de pression et des intensités. Quelques résultats typiques des essais réalisés sur cette installation sont présentés dans les figures suivantes.
Les niveaux de pressions relevés au point A pour une vitesse de rotation de la pompe de $1200 \mathrm{tr} / \mathrm{mn}$ ainsi que les niveaux d'intensité calculés sont tracés dans la figure 7. (Dans cette représentation de l'intensité la moitié supérieure du graphique correspond à un flux d'énergie "positif" (pompe $\rightarrow$ vanne) et la moitié inférieure à un flux "négatif" (vanne $\rightarrow$ pompe).

Alors que le spectre de pression ne fournit que très difficilement des renseignements sur le ou les origines du bruit, le spectre d'intensité permet d'identifier directement les sources: dans l'ensemble le flux d'énergie est négatif et provient par conséquent de la vanne. L'énergie émise par la pompe est concentrée autour de quelques fréquences qui sont des harmoniques de la fréquence de passage des aubes $(100,200,300,400 \mathrm{~Hz})$. Elle se manifeste par des raies positives qui émergent très nettement du spectre.

Dans la figure 8 sont tracées les mêmes courbes pour la vitesse de $2700 \mathrm{tr} / \mathrm{mm}$. Lorsque la pompe monte en régime, les niveaux de pression augmentent aussi mais sans que la forme du spectre laisse apparaitre des modi. fications dans les rapports entre pompe et vanne. Par contre, le spectre de l'intensité montre que les flux d'énergie sont maintenant très différents: les raies dues a la pompe ont glissé vers des fréquences plus élevées $(225,450,675,900 \mathrm{~Hz})$. En basse fréquence $(f<500 \mathrm{~Hz})$
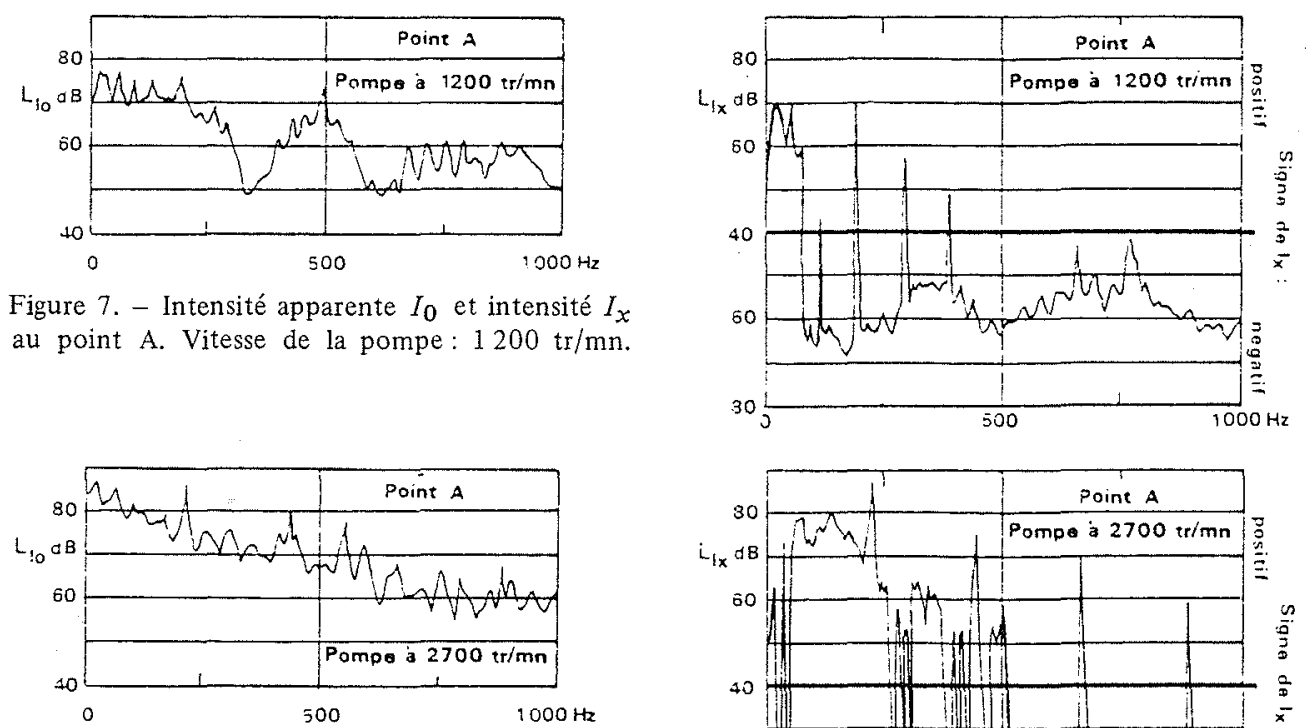

Figure 8. - Intensité apparente $I_{0}$ et intensité $I_{x}$ au point $A$. Vitesse de la pompe : $2700 \mathrm{tr} / \mathrm{mn}$.

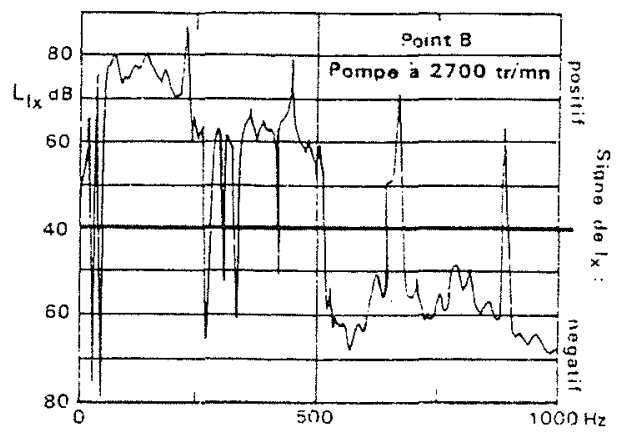

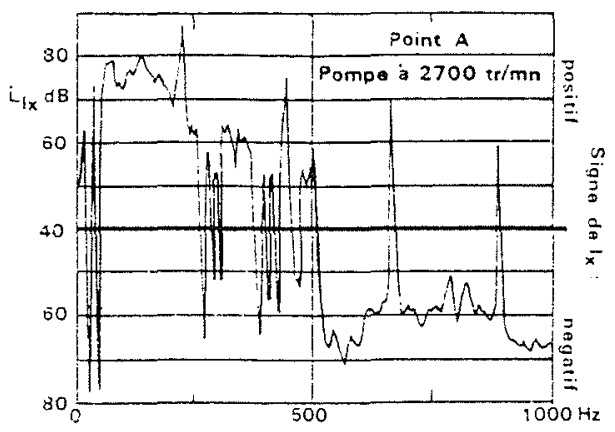

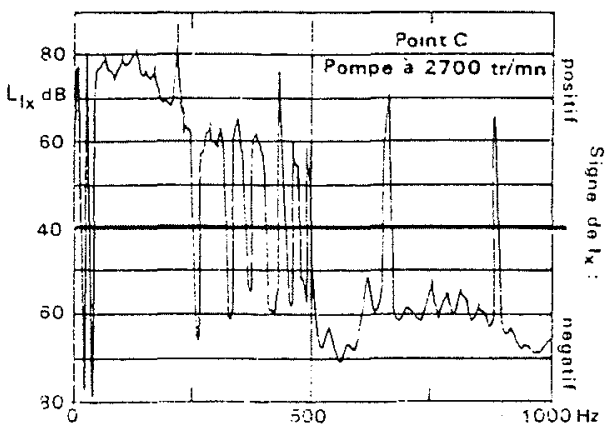

Figure 9. - Intensités $I_{x}$ aux points B et C. Vitesse de la pompe : $2700 \mathrm{tr} / \mathrm{mn}$. 

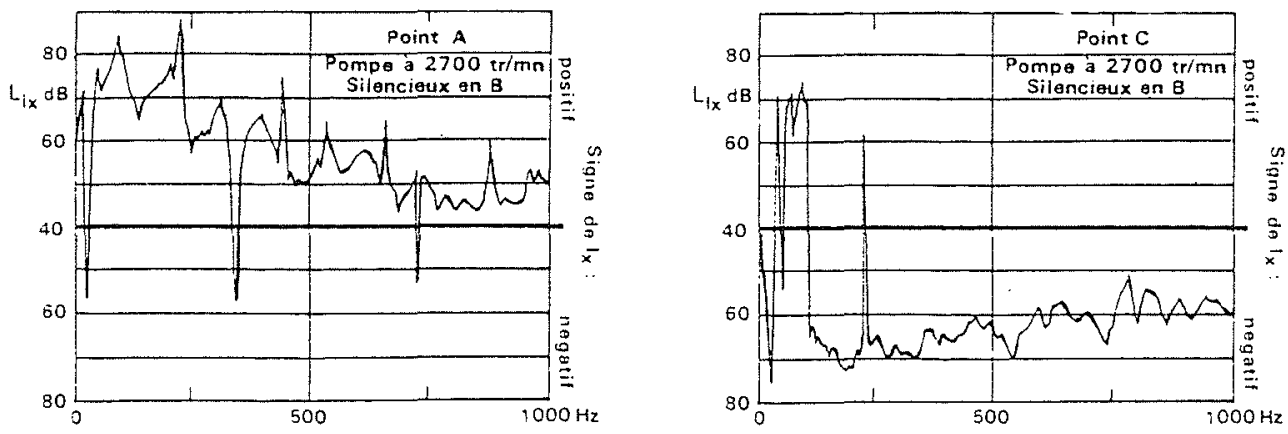

Figure 10. - Intensités $I_{x}$ aux points A et C lorsqu'on dispose un silencieux en B. Vitesse de la pompe : $2700 \mathrm{tr} / \mathrm{mn}$.

la pompe émet en outre un bruit large bande dont l'énergie est supérieure à celle de la vanne, entrainant ainsi un flux positif. Au-dessus de $500 \mathrm{~Hz}$, le flux négatif provient toujours de la vanne avec un spectre dont l'allure a peu changé par rapport au régime $1200 \mathrm{tr} / \mathrm{mn}$.

La figure 9 montre pour le même régime les intensités $I_{x}$ déterminées aux points $\mathrm{B}$ et $\mathrm{C}$. On constate alors que les différences entre les résultats obtenus à différents endroits du conduit ( $A, B$ et $C$ ) sont insignifiantes.

Pour prouver d'une façon pratique que l'étude du signe de l'intensité permet de séparer effectivement les deux sources, nous les avons isolées acoustiquement aussi bien que possible par un silencieux (filtre acoustique à parois souples) placé au point $\mathrm{B}$. Puis nous avons déterminé de nouveau les intensités aux points $A$ et $C$ : figure 10 . Le spectre d'intensité au point $A$ ne montre pratiquement plus de flux négatif, le silencieux ayant absorbé l'énergie émise par la vanne. Au point $\mathrm{C}$ au contraire, le flux est essentiellement négatif, la contribution de la pompe étant supprimée (sauf en très basse fréquence où l'efficacité du silencieux est faible).

\section{Conclusions}

Une des applications de l'intensimétrie est son utilisation pour l'étude des problèmes acoustiques dans les circuits hydrauliques et aéroliques. La théorie montre qu'elle permet de caractériser complètement une onde plane quasi-stationnaire dans un conduit. Les grandeurs qui doivent être mesurées sont -- comme dans le cas plus général-- les pressions acoustiques en deux points du conduit et le déphasage entre les deux signaux. (Dans le cas d'un bruit large bande, le déphasage est obtenu par la détermination simultanée des transformatées de Fourier des signaux de pression)
A partir de ces trois informations, on peut obtenir: - une mesure du vecteur intensité $I$ qui caractérise le flux d'énergie global dans une section et notamment le sens de sa propagation. Cette mesure a de plus l'intérêt d'être théoriquement indépendante de la position le long du conduit ;

- le taux d'onde progressive $\beta$ qui est le rapport entre minima et maxima de pression. La connaissance de ces deux grandeurs $\vec{I}$ et $\beta$ permet de déterminer les minima et maxima de la pression ainsi que sa valeur moyenne tout le long du conduit;

- la distance entre point de mesure et le réflecteur qui est la cause des ondes quasi-stationnaires.

Des expériences de principe, réalisées en son pur et en bruit large bande, dans un tube de Kundt et dans deux circuits hydrauliques de dimensions différentes ont confirmé ces possibilités.

\section{Bibliographie}

[1] PAVIC G. - Measurement of Sound Intensity, ISV (avril 1977) $51(4), 533-545$.

[2] FAHY F.J. - A Technique for Measuring Sound Intensity with a Sound Level Meter. Noise Control Eng. (nov,déc. 1977) 9 (3), 155-162.

[3] LAMBERT J.M. et al. - La mesure directe de l'intensité acoustique, CETIM - Informations (décembre 1977) $53,78-91$.

[4]CHUNG J.Y. et al. - Practical Measurement of Acoustic Intensity, the Two-Microphone Cross-Spectral Method, Proceedings of Inter-Noise 78, 893-900.

[5] HAMAN M. et al. - Untersuchungen zur Ortung von Lärmschallquellen an Maschinen unter Berücksichtigung des Nahfeldeffekts. Proceedings of DAGA 76, 343-346.

[6] CHUNG J.Y. et al. - Transfer Function Method of Measuring In-duct Acoustic Properties, JASA (septembre 1980) $68,907-921$. 